\title{
A COCOON TRANSPARENCY AND THE 3C 345 LOW FREQUENCY VARIABILITY
}

\author{
L.I.MATVEENKO \\ Space Research Institute of RAS, Profsojuznaja 84/32, \\ 117810 Moscow, Russia
}

\begin{abstract}
The structure of the quasar $3 \mathrm{C} 345$ is studied at $\lambda=49 \mathrm{~cm}$. The core has a low-frequency cut off spectrum and $\alpha \sim 3$. The brightest knot is in the nearest part of the jet. The cocoon wall absorbs low-frequency emission and changes the polarization orientation, $\mathrm{RM}=3500 \mathrm{rad} \mathrm{m} \mathrm{m}^{-2}$, $B_{\|} \sim 100 \mu \mathrm{G}$ at the core region.
\end{abstract}

The studies of the quasar $3 \mathrm{C} 345$ fine structure at $\lambda=49 \mathrm{~cm}$ with a global VLBI network show that the core emission is weak $[1,2]$. The core spectrum has a low frequency cut off and the spectral index in the optically thick part is $\alpha \sim 3$. The compact brightest component corresponds the nearest part of the jet, with a size of $\sim 5 \times 4$ mas. In 1983.9-1990.8 the flux density of the component and the solid angle increased by a factor of $\sim$ 2. The brightness temperature was $T_{b}=0.5 \cdot 10^{12} \mathrm{~K}$ and did not change significatively.

According to the black hole model, an accretion disk implies a surrounding medium in the azimuth plane, leaving a relatively free space in the direction of the rotation axis. The relativistic plasma is ejected along the axis, within an angle of $\leq 1 s t r$ [3]. A magnetic field focuses the plasma into thin filaments. The rotation of the black hole (the ejector) twists the filaments around the axis $[4,5]$ and forms the spiral structure jet [6-8].

The ejector is the source of the synchrotron emission, i.e. what we call the "core". The is core located $\sim 15$ mas $\mathrm{E}$ of the brightness peak in the $\lambda=49 \mathrm{~cm}$ map $[1,2]$ and has a brightness temperature of $T_{b} \sim 10^{10} \mathrm{~K}$. The relativistic plasma flow is surrounded by thermal plasma in the form of a cocoon. The cocoon absorption of the core emission at $\lambda=49 \mathrm{~cm}$ is equal $\sim 100$ and the optical depth $\tau \sim 5$. The absorption at $6 \mathrm{~cm}$ will be $\leq 20 \%$ and the cocoon wall is practically transparent at $\mathrm{mm}-\mathrm{cm}$ wavelengths. 
The time scale of the low frequency variability is $t \sim 1 \mathrm{yr}$ [9] and the recombination time of the thermal plasma must be $\leq 1 \mathrm{yr}$. The recombination time is equal $t_{r}=10^{5} N_{e}^{-5} \mathrm{yr}$ therefore $N_{e} \sim 10^{5}$. If the optical depth of the cocoon wall is $\tau \sim 1$, the wall thickness is $l \sim 10^{-3} p c$. The thickness increases with the distance from the core and the total number of thermal electrons in a column is $N_{e} \sim r^{-2}$. The transparency is determined by the emission measure $\mathrm{EM} \sim N_{e}^{2} l$ and varies as $\sim r^{-4}$. The rotation measure $R M \sim N_{e} B_{\|} l$ varies as $\sim r^{-3}$.

The wall is transparent at the centimetre wavelengths, however, the screen can change the orientation of the polarization plane. According to VLBA measurements of $3 \mathrm{C} 345$ with a beam size $\sim 5$ mas [10], the rotation measure in the $18-21 \mathrm{~cm}$ band is $R M \sim 28 \mathrm{rad} \mathrm{m}^{-2}$ and the degree of polarization is $\sim 4 \%$. The size of polarized region is equal $\sim 5$ mas [11]. The brightest emission region at $18 \mathrm{~cm}$ corresponds to the nearest part of the jet $[1,2]$. We propose that the polarization emission at $18 \mathrm{~cm}$ arises from the same region. The polarization position angle, corrected for the Faraday rotation, gives the position angle of the magnetic field and the jet orientation at the distance of $\sim 5$ mas from the core. The rotation measure, $\mathrm{RM} \sim r^{-3}$, will be $3500 \mathrm{rad} \mathrm{m}^{-2}$ at the core region and the magnetic field is $B_{\|} \sim 100 \mu \mathrm{G}$.

The changes in the cocoon wall transparency will change the polarization position angle and the low frequency emission.

This work supported by the Soros's ISF, Grant MFR300.

\section{References}

[1] Matveenko, L.I., Graham, D.A., Pauliny-Toth, I.I.K., et al. (1992) Pis'ma v Astron. Zh.,Vol. 18,p 931

[2] Matveenko, L.I., Pauliny-Toth, I.I.K., Baath, L.B., et al. (1996) Pis'ma v Astron. Zh., Vol. 22,p in press

[3] Begelman, M.C., Blanford, R.D. Rees, M.J. (1981) Rev.Mod.Phys., Vol. 56,p 255

[4] Shakura, N.I. and Sunyaev, R.A. (1973) $A \& A$,Vol. 24,p 337

[5] Lovelace, R.V. and Berk, H.L. (1991) Astroph. J.,Vol. 379,p 695

[6] Unwin, S.C. and Wehrle, A. (1992) Astroph.J.,Vol. 398,p 74

[7] Krichbaum, T.P., Witzel, A., Graham, D.A.,et al. (1993) $A \& A$, Vol. 275,p 375

[8] Zensus, J.A., Cohen, M.H., and Unwin, S.C. (1995) Astroph. J., Vol. 443,p 35

[9] Padrielli, L., Eastman, W., Gregorini, L., et al. (1991) $A \& A$, Vol. 249, p 351

[10] Rudnick, L., and Jones, T.W. (1983) Astron.J.,Vol. 89,p 518

[11] Browne, L.F., Roberts, D.H., Wardle, J.F.C. (1994) Astroph. J., Vol. 437,p 108 